\title{
Modeling Sympatric Speciation in Quasiperiodic Environments
}

\author{
Jasmine Foo, Cymra Haskell, Natalia L. Komarova, Rebecca A. Segal, \\ and Karen E. Wood
}

\begin{abstract}
Sympatric speciation is the emergence of new species from a single ancestral species while inhabiting the same geographic region. This process presents an interesting problem for theoretical studies of evolution. One mechanism by which sympatric speciation might occur is periodic or quasiperiodic fluctuations in the abundance of the resources. In this paper inspired by the experimental findings of (Herron and Doebeli, PLoS Biol. 11, p. e1001490, 2013), we present a number of models of asexual speciation of $E$. coli, which range in the level of biological detail and the degree of analytical treatment. We show that coexistence of multiple species arises as a robust phenomenon, even in the presence of spatial and temporal randomness.
\end{abstract}

Keywords Periodic forcing - Bacterial evolution - Fast switchers - Slow switchers • Ecological modeling • Seasonal changes • Diversity

AMS(MOS) subject classifications. 92D15, 34C60, 34F05

\author{
J. Foo $(\square)$ \\ School of Mathematics, University of Minnesota, 206 Church St SE, Minneapolis, \\ MN 55455, USA \\ e-mail: jyfoo@umn.edu \\ C. Haskell \\ Department of Mathematics, University of Southern California, 3620 S. Vermont Ave, \\ Los Angeles, CA 90089, USA \\ e-mail: chaskell@usc.edu \\ N.L. Komarova $(\bowtie)$ \\ Department of Mathematics and Department of Ecology \& Evolutionary Biology, University \\ of California Irvine, Irvine, CA 92697, USA \\ e-mail: komarova@uci.edu

\section{R.A. Segal} \\ Department of Mathematics and Applied Mathematics, Virginia Commonwealth University, \\ Richmond, VA 23284, USA \\ e-mail: rasegal@vcu.edu \\ K.E. Wood \\ Department of Mathematics, University of California Irvine, Irvine, CA 92697, USA \\ e-mail: kewood@uci.edu




\section{Introduction}

Understanding mechanisms of speciation has long been considered one of the fundamental problems in biology. Four geographic modes of speciation have been identified based on the extent to which speciating populations are isolated from one another: allopatric, peripatric, parapatric, and sympatric. Arguably the most puzzling mode of speciation is sympatric speciation, where new species evolve from a single ancestral species while inhabiting the same geographic region. In the literature, many aspects of sympatric speciation have been studied theoretically, see, e.g., [3, 9, 18, 19, 22, 23]. The theory of evolutionary branching explains the dynamics of species emergence and has been applied to both sexual [5] and asexual populations $[8,11]$.

In [7], the power of this theory was demonstrated by using several well-known ecological models, including models for symmetric and asymmetric competition, mutualism, and predator-prey interactions. Evolutionary branching occurs when frequency-dependent selection splits a phenotypically monomorphic population into two distinct phenotypic clusters. For this mechanism to work, selection must drive the population toward a fitness minimum in phenotype space. Conditions have been established that allow for evolutionary branching in different models. For more details on the theory of adaptive dynamics, see [6]; for a discussion of this theory and its contributions to our understanding of sympatric speciation, see $[12,27]$.

To obtain a theoretical understanding of sympatric speciation, two different issues must be addressed. (i) Reproductive isolation: How can a population split into two sub-species in the presence of recombination, which leads to the production of intermediate offspring? (ii) Coexistence: How can multiple diverging lineages coexist in sympatry? It is the second issue that we will focus on in this paper.

A fascinating case study that motivated our work was the paper [13]. By using a model system of evolving bacteria Escherichia coli (E. coli), the authors demonstrated that speciation can be observed in a predictable, reproducible fashion. They documented the genetic basis and the evolutionary dynamics of adaptive diversification, and showed that in several replicates of the experiment, parallel genetic changes underlie similar phenotypes in independently evolved lineages. The experimental system used by [13] involved E. coli competing for two carbon sources, which were replenished daily and depleted by the bacteria over the course of each day. The presence of two periodically renewed food sources caused initially isogenic populations of $E$. coli to diversify into two coexisting phenotypes, which corresponded to two different physiological adaptations in the carbohydrate metabolism.

The idea that seasonally changing environments can create diversity has been developed in the literature for several decades, see, e.g., [1, 4, 15, 16, 20, 21, 26]. [16] proposed that the co-existence of many different species of phytoplankton can be explained by temporal changes in the environment that preclude an equilibrium from being achieved under a given set of environmental conditions. This means that the principle of competitive exclusion [2] does not apply. In [26], the authors created and 
analyzed an elegant model where a number of species consume different resources at different intensity, and the resources are replenished constantly. Paper [25] emphasized the fact that frequency dependent competition allows for stable species coexistence in seasonally changing environments. Such frequency dependence can occur, for example, when variation in resource availability is generated through depletion and subsequent replenishment of these resources. By using the E. coli system, the authors showed that removing "seasonality" destroys coexistence and leads to dominance of a single species.

In the present paper, as in [17], we treat the coexistence problem of sympatric speciation as an ecological question, where the issue of reproductive isolation is of secondary importance. Therefore, we restrict our attention to evolving asexual populations (this assumption is natural for the case study of E. coli). We develop a number of models inspired by the $E$. coli evolution in the experiments of $[13,25]$. We start from a model that is case-study based and specific to the $E$. coli experiment, and then create a more abstract model which only keeps the components essential for speciation driven by a periodically changing environment. We show that if different species can consume the same resources and even have the same preferred resource, and only differ by their switching behavior when the preferred resource becomes sparse, "seasonal" changes in the abundance of the resources can drive speciation. Among the different phenotypes, the most "different" ones will survive and achieve quasi-stable coexistence. This result continues to hold in quasiperiodic random environments where the time period between resource replenishment and the amount of resources replenished varies stochastically. Further, it continues to hold in spatially distributed systems.

The rest of this paper is organized as follows. In section 2 we will develop a mathematical model that describes speciation in the experiments of [13]. In section 3 we create a more abstract model, which captures the essential components of the case study while omitting details that are not necessary for speciation. In this model, speciation is driven by the periodically changing environment. Both analytical and numerical results are presented for periodically changing environments. We further depart from the assumption of periodicity and investigate speciation in quasiperiodic and random environments. Section 4 extends the discussion to spatially distributed systems. Conclusions are presented in section 5 .

\section{Speciation in E. coli: A Case Study}

It has been shown in $[10,13]$ that sympatric diversification can occur with frequency-dependent selection that arises as a result of cooperation, competition, or predation. In this case study, we develop a mathematical model of an experiment conducted by Herron and Doebeli in which sympatric diversification appears to occur through the interaction of two divergent phenotypes with the environment. In a periodically replenished environment, a phenotype of E. coli emerges that modifies 
the environment creating a niche in which a second phenotype can flourish. This environmental feedback produces the frequency-dependent selection that is central to theories of sympatric diversification.

\subsection{Biological Background}

Herron and Doebeli cultivated three parallel populations of E. coli, each of which was founded from an isogenic line and cultured in well-mixed conditions for 183 days (about 1230 generations) in a medium of glucose and acetate. Each day the bacteria used up all the available glucose and then underwent what is known as a diauxic switch to acetate consumption before entering a stationary phase. At the end of the day they were transferred to a fresh medium of glucose and acetate. In each of the three populations, two phenotypes emerged that coexisted for the duration of the experiment. The phenotypes were distinguished by their diauxic resource use. The fast switchers (FS) exhibited slower growth when glucose was abundant, but switched to acetate consumption even while glucose was still in abundance. The slow switchers (SS) exhibited faster growth when glucose was abundant, but took longer to switch to acetate consumption when the glucose was depleted.

In the presence of multiple nutrients, bacteria have complex and diverse regulatory mechanisms enabling them to preferentially utilize those nutrients that facilitate the fastest growth. See [29] for a thorough review of the current understanding of the metabolic processes involved in glucose and acetate consumption and in the switch from the former to the latter. The following is a simplified biological model of these processes.

When metabolizing glucose, cells take up glucose from their environment and break it down through glycolysis to pyruvate and then acetyl-CoA producing ATP in the process. The acetyl-CoA is broken down into carbon dioxide by the TCA cycle, converted to acetate by the PTA-ACKA pathway, and converted to other compounds such as ethanol by other pathways. The first two processes produce ATP. The production or dissimilation of acetate has the effect of stimulating glycolysis by re-oxidizing $\mathrm{NADH}$ and thereby replenishing $\mathrm{NAD}^{+}$required by glycolysis and recycling the coenzyme A required to convert pyruvate to acetyl-CoA [29]. This is illustrated in figure 1(b). When metabolizing or assimilating acetate, the PTAACKA pathway reverses; cells take up acetate and convert it to acetyl-CoA using ATP in the process. The acetyl-CoA is then broken down into carbon dioxide by the TCA cycle releasing ATP. This is illustrated in figure 1(a).

A warm, alkaline, anaerobic, and nutrient-rich environment favors acetate dissimilation and a cool, acidic, aerobic, and acetate-rich environment favors acetate assimilation. Thus, in the presence of abundant glucose, wild type cells dissimilate acetate, thereby stimulating glycolysis. As the acetate concentration increases, the acidity increases and as the glucose is depleted, the growth rate declines increasing the oxidation of the medium. The result is that the PTA-ACKA pathway reverses direction and the cells start to assimilate acetate. This, in turn, results in a lower level of assimilation of glucose. This is known as the acetate switch. 
a
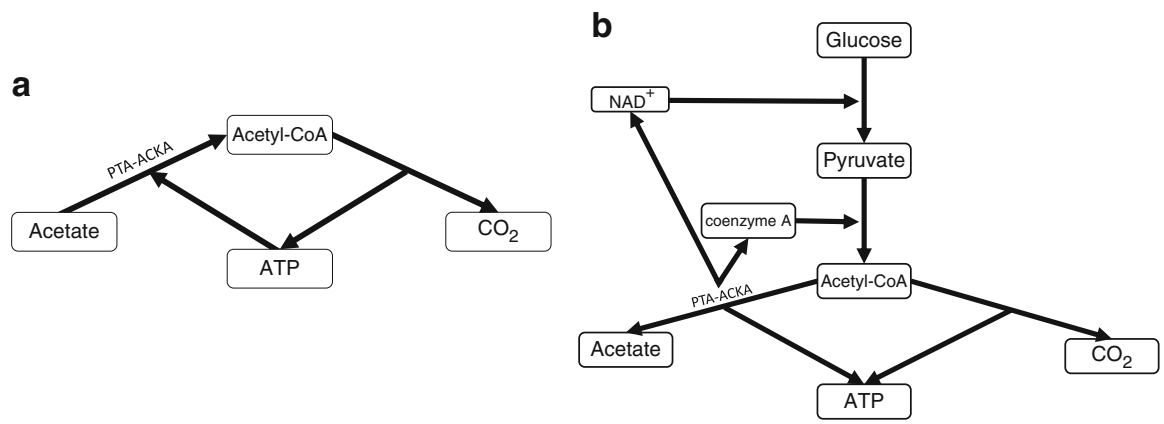

Fig. 1 A schematic illustrating the biological processes involved in (a) acetate assimilation and (b) dissimilation.

\subsection{Modeling E. coli}

Our model admits any number $n$ of phenotypes of $E$. coli. Let $g, a$, and $x_{i}, 1 \leq i \leq n$ denote the concentrations of glucose, acetate, and the $i$ 'th phenotype, respectively, in solution.

Experimental studies of Spencer, Bertrand, Travisano, and Doebeli [24] indicate that the slow switchers in the Herron-Doebeli experiment behave similarly to the wild type cells while the fast switchers have one or more mutations that repress acetate dissimilation. In light of this, the different phenotypes of E. coli are distinguished in our mathematical model by the level of glucose that stimulates the activity level of the PTA-ACKA acetate dissimilation pathway. More specifically, the variable $l_{i}$ denotes the fraction of metabolized glucose that is dissimilated as acetate in the $i$ 'th phenotype. We suppose $l_{i}$ depends on $g$. The functional form of $l_{i}(g)$ is the same for all phenotypes; there is a threshold level of glucose below which $l_{i}$ decays exponentially to 0 and above which it increases exponentially to 1. Moreover, the exponential rate of increase and decrease $\tau$ is the same for all phenotypes. It is the threshold $g_{i}$ that differs between phenotypes; for the fast switchers $g_{i}$ is large since they only dissimilate acetate when the glucose level is very high and for the slow switchers $g_{i}$ is small causing them to switch from acetate dissimilation to acetate assimilation only when the glucose level drops below some relatively small level.

When a cell is assimilating acetate it dissimilates less acetate and thereby reduces its stimulation of glucose assimilation. For this reason the value of $l_{i}$ affects the following rates in our mathematical model;

a) the rate acetate is dissimilated per molecule of glucose assimilated,

b) the rate acetate is assimilated, and

c) the rate glucose is assimilated.

At low concentrations of glucose, each cell consumes glucose at a rate that is proportional to $l_{i} g$, but this rate levels off to a maximum $l_{i} \sigma$ at high concentrations. 
Similarly, at low concentrations of acetate, each cell consumes acetate at a rate that is proportional to $\left(1-l_{i}\right) a$ but this levels off to a maximum $\left(1-l_{i}\right) \rho$ at high concentrations. We model these rates with the functions

$$
l_{i} \sigma \frac{g}{K_{g}+g} \quad \text { and } \quad\left(1-l_{i}\right) \rho \frac{a}{K_{a}+a}
$$

respectively. Let $\gamma$ and $\alpha$ denote the growth rates of the cells due to consumption of glucose and acetate, respectively, and $\delta$ the natural death rate of the cells. Let $\beta$ denote the acetate excreted per molecule of glucose due to acetate dissimilation. Since the value of $l_{i}$ denotes the fraction of metabolized glucose that is dissimilated as acetate, the rate of increase of acetate due to acetate dissimilation is equal to the product of $l_{i}, \beta$ and the rate at which glucose is metabolized. Table 1 summarizes these parameters and their roles in the model.

This gives us the following system of differential equations modeling the populations in the experiment:

$$
\begin{aligned}
& \dot{l}_{i}=\tau\left[H\left(g-g_{i}\right)-l_{i}\right] \\
& \dot{x}_{i}=\gamma \frac{l_{i} \sigma g}{K_{g}+g} x_{i}+\alpha \frac{\left(1-l_{i}\right) \rho a}{K_{a}+a} x_{i}-\delta x_{i} \\
& \dot{g}=-\sum_{i=1}^{n} \frac{l_{i} \sigma g}{K_{g}+g} x_{i} \\
& \dot{a}=\beta l_{i} \sum_{i=1}^{n} \frac{l_{i} \sigma g}{K_{g}+g} x_{i}-\sum_{i=1}^{n} \frac{\left(1-l_{i}\right) \rho a}{K_{a}+a} x_{i},
\end{aligned}
$$

where the function $H$ is the Heaviside function. System (2.1-2.4) represents the dynamics observed during a single day of $E$. coli experiments. To study the evolutionary dynamics, we incorporate the resampling procedure in the following way. We simulate equations $(2.1-2.4)$ for a certain duration of time $(\bar{t}=24)$. Then, we re-normalize the population sizes $x_{i}$, so that their sum is equal to 1 , and reset variable $g$ and $a$ to their initial values. These new values serve as the initial conditions for the next iteration. The simulation shown in Figure 2(a) shows that this model admits the possibility of coexistence with two phenotypes; a slow

Table 1 Parameters used in (2.1)-(2.4) and their definitions.

\begin{tabular}{l|l}
\hline Parameters & Biological roles \\
\hline$\gamma$ & cell growth rate due to glucose consumption \\
\hline$\alpha$ & cell growth rate due to acetate consumption \\
\hline$\beta$ & acetate excreted per molecule of glucose \\
\hline$g_{i}$ & glucose switching threshold for $i$-th phenotype \\
\hline$\rho, K_{a}$ & control maximum acetate consumption rate per cell \\
\hline$\sigma, K_{g}$ & control maximum glucose consumption rate per cell \\
\hline
\end{tabular}



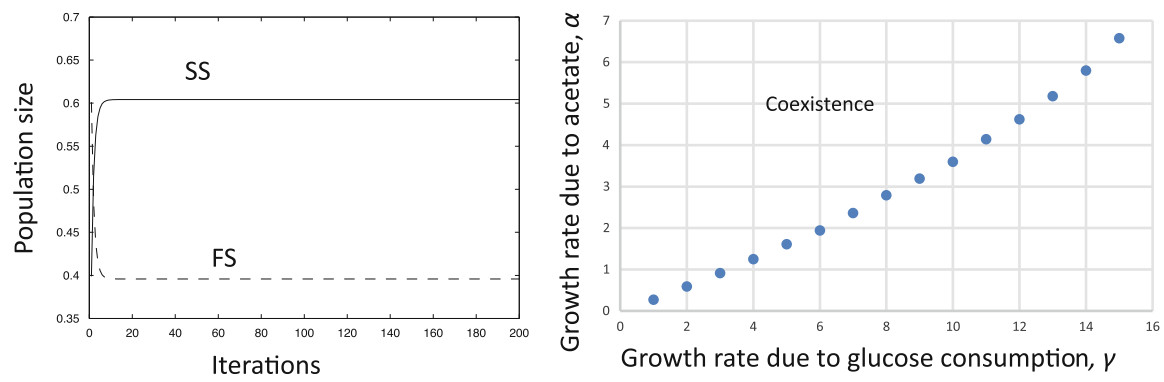

Fig. 2 Simulations showing the coexistence behavior of system (2.1-2.4). (a) Graph of the levels of SS and FS E. coli, using initial values of glucose and acetate of 1 and initial amounts of SS and FS of .1 and .9, respectively. The two phenotypes have $g_{1}=.01$ and $g_{2}=10$, where $g_{1}$ and $g_{2}$ are the thresholds for switching from glucose to acetate for SS and FS, respectively. The remaining parameters are $\gamma=12, \alpha=8, \sigma=1, \rho=1, K_{g}=1, K_{a}=1, \beta=2, \delta=0.1, \tau=2$. (b) A numerical study of coexistence in the $(\gamma, \alpha)$ parameter space. The rest of the parameters are the same as in (a).

switcher and a fast switcher that always dissimilates acetate. This simulation shows 200 replenishments of resources at time-intervals of length $\bar{t}$.

Figure 2(a) presents a more systematic study of coexistence in this model. The region of coexistence was found by examining the simulations as $\alpha$ and $\gamma$ were varied (while the rest of the parameters were fixed). Simulations were run where $\gamma$ was held constant as $\alpha$ was varied at .01 increments from 0 . The code checked for stability of the solution by comparing the population of the cell types across 100 resource replenishments. If the difference between the population at the end of the two replenishment time periods was not small enough, the simulation would be run for another 400 replenishments, and checked again. If the required threshold was met, then the populations were checked for positivity. If the populations were not both positive, $\alpha$ was increased by .01, and the simulations began again. If the positivity requirement was passed by both populations, the $\gamma$ and $\alpha$ values were recorded, $\gamma$ was increased by 1 and the test was started from the beginning.

The case study model (2.1-2.4) will serve as a foundation for building an autonomous model that exhibits experimental behavior and at the same time is amenable to mathematical analysis, see the next section. An alternative mathematical description of the E. coli experiments was developed by [10]. The main difference between system (2.1-2.4) and the model in [10] is the different mathematical techniques used to describe the metabolic switch. In [10], the switch between the two consumption behaviors happens as a function of the physical time that has elapsed from the beginning of a cycle. In our model, we explicitly assess the level of the glucose, see equation (2.1). While neither our model nor that of [10] allow an analytical description, in the next section we develop a simplified version that turns out to be amenable to analysis. The simplified model is based on our modeling choice expressed in equation (2.1). 


\section{A General Model of Speciation in a Periodic Environment in the Presence of Two Food Sources}

In the specific models of speciation describing the experiments with $E$. coli, we were able to observe speciation under a wide range of parameters. It can also be noted that several changes in the model structure are still compatible with stable coexistence of two multiple phenotypes in the system. In this section we will formulate a simpler and less specific model that is easier to understand analytically.

\subsection{Model Formulation}

In order to create a concise model which captures the essential features of speciation mechanisms observed in the experiments by [13], we can assume a reductionist approach and omit several aspects of the model that are not necessary for speciation. In particular,

- The resampling where the new initial condition normalizes the populations of the different organisms can be omitted, such that only the food sources are added (quasi)periodically.

- The functional forms describing the saturation in terms of the food source can be omitted. In this setting, the amounts of nutrients do not grow and only decay in the course of each day, and therefore there is no danger of an unlimited increase of nutrient uptake.

- The food supplies do not have to be produced in the metabolic process.

- The switch from one mode of consumption to the other does not have to happen as a step function. It can be a more gradual process, to keep the numerics stable and robust to rounding errors.

While dropping these details, we must keep the essential features of the system:

1. There are two food sources, $g$ and $a$, with one of them (variable $g$ ) being more energy rich. The food sources are replenished at the beginning of each day. In the course of the day, they are consumed by the different species.

2. While all phenotypes prefer to feed on source $g$, they are able to consume both food sources in different proportions.

3. Different phenotypes tend to switch from predominantly consuming $g$ to predominantly consuming $a$. The switching is triggered by the lowering of the amount of $g$.

4. The phenotypes differ from each other by the way this switch happens.

The following non-dimensionalized system includes these assumptions:

$$
\dot{x}_{i}=\left[S_{i}(g) g \gamma+\left(1-S_{i}(g)\right) a \alpha-d\right] x_{i}, \quad 1 \leq i \leq n,
$$




$$
\begin{aligned}
& \dot{g}=-\sum_{i=1}^{n} S_{i}(g) g x_{i}, \\
& \dot{a}=-\sum_{i=1}^{n}\left(1-S_{i}(g)\right) a x_{i},
\end{aligned}
$$

where the initial conditions are given by

$$
x_{i}(0)=x_{i 0}, \quad g(0)=g_{0}, \quad a(0)=a_{0} .
$$

In this system, we assume that each phenotype at all times consumes $g$ and $a$ with proportions $S_{i}(g)$ and $\left(1-S_{i}(g)\right)$ respectively (that is, the total consumption is always 1$)$. The consumption happens at a rate proportional to the abundance of each food source and the resulting growth is proportional to its energetic efficiency, $\gamma$ and $\alpha$.

The fraction of effort to consume $g$ depends on its abundance according to the function $S_{i}(g)$, where $0 \leq S \leq 1 . S_{i}$ is a smooth step-like function of a finite width, with the switch around the value $g_{i}$. For example, we can use

$$
S_{i}(g)=\frac{g^{k}}{g^{k}+g_{i}^{k}},
$$

where $k$ measures the steepness of the switch (e.g., $k=10)$.

Parameters of the model are defined in Table 2. Note that the parameters (tables 1 and 2) do not have the same mathematical meaning in models (2.1-2.4) and (3.1-3.3). Some parameters are denoted by the same symbols in the two models, because of their similar biological meaning. For example, $\alpha$ and $\gamma$ stand, respectively, for the growth rates of cells due to the consumption of acetate and glucose. The units however are not the same because of the structural differences between the models. Model (2.1-2.4) includes saturation in the amount of food source, as well as a separate dynamic variable, $l_{i}$, which is the activity level of the acetate dissimilation pathway. These layers of complexity are removed in model (3.1-3.3). Therefore, the correspondence between the variables denoted by the same symbol is not literal.

Table 2 Parameters used in (3.1)-(3.3) and their definitions.

\begin{tabular}{l|l}
\hline Parameters & Biological roles \\
\hline$\gamma$ & cell energetic efficiency on glucose \\
\hline$\alpha$ & cell energetic efficiency on acetate \\
\hline$d$ & cell death rate \\
\hline$g_{i}$ & glucose switching threshold for $i$-th phenotype \\
\hline$k$ & steepness parameter for switch (used in $\left.S_{i}(g)\right)$ \\
\hline
\end{tabular}


We further note that model (3.1-3.3) is similar to the model considered in [26], where interspecific competition for shared resources is studied. However, in the model of [26], the uptake rate of each resource is solely a function of the abundance of that resource. In contrast, here in order to describe the switch to acetate consumption triggered by glucose levels, we must consider the uptake rate of acetate as a function of both the acetate and glucose abundance.

System (3.1-3.3) is iterated in the following manner. The ODEs are solved on the interval $0 \leq t \leq \bar{t}$, where $\bar{t}$ is the time of resampling. Then, the new initial conditions for the phenotype abundances are set to be $x_{i 0}=x_{i}(\bar{t})$, that is, the abundances remain the same, and the food sources are reset to the values $g_{0}$ and $a_{0}$. The solution procedure is again repeated for each iteration.

Figure 3 shows the typical dynamics of system (3.1-3.3). 20 types were randomly created by assigning their value $g_{i}$ from a uniform distribution between 0 and 0.1 . The initial value of one of the types was assumed larger than that of the rest, to mimic the dynamics presented in Figure 2 of [13]. After 2000 iterations, three phenotypes emerged. The panel on the right shows the abundance of each of the 20 types after 2000 iterations (blue) together with the corresponding values of $g_{i}$ for each type. The most abundant phenotype (which usually expands first) corresponds to the slowest switcher (its threshold value is the lowest of the set). There can be one or more types that coexist with this SS type on a long term. These are characterized by a large value of $g_{i}$, so these are FS. The initial condition, where one of the types is significantly more abundant, is not necessary to observe coexistence.

More than one FS types can coexist for a long time (and sometimes only one remains). In reality this corresponds to the heterogeneity of types that can be roughly classified as one type.

a

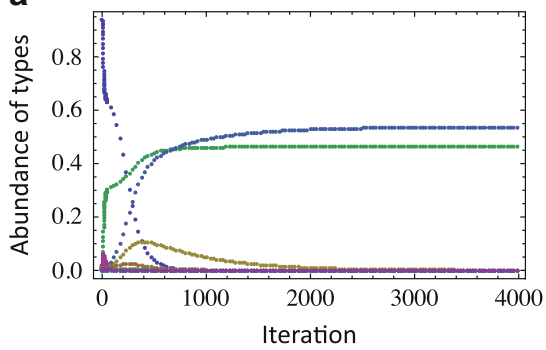

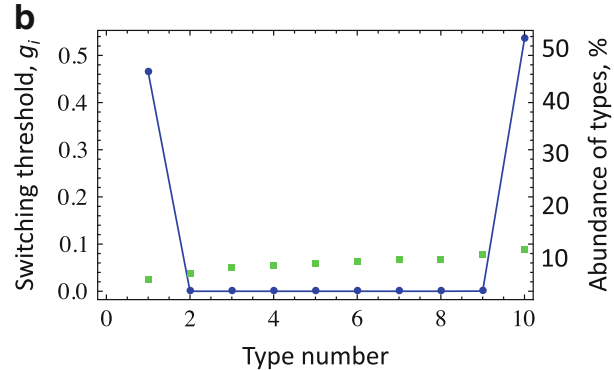

Fig. 3 Typical dynamics of system (3.1-3.3), where 10 types were randomly created by assigning their value $g_{i}$ from a uniform distribution between 0 and 0.1 . In (a), the relative abundances of all types are shown as a function of the iteration number. In (b), the abundances at iteration 2000 are shown in blue, together with the values $g_{i}$ for each type (green), ordered in terms of their threshold value. The parameters of the system are: $n=10, \bar{t}=20$ (resampling time), $a_{0}=g_{0}=1$ (the initial values of $a$ and $g$ at the start of each iteration), $k=10, \gamma=1, \alpha=0.1, d=0.1$. 


\subsection{Stability Properties of the Single Period Map}

Let $\left\{x_{i}\right\}_{i=1}^{n}, g$, and $a$ satisfy the system (3.1)-(3.3) for $t \in[0, \bar{t}]$. We make the simplification that the functions $S_{i}$ are simply the analogous Heaviside function in this section. Define the forward evolution map under the system (3.1)-(3.3) by $\psi_{t}\left(\mathbf{x}_{0}\right)$, where $\mathbf{x}_{0}=\left(x_{1}(0), \ldots, x_{n}(0)\right)$ is the initial abundances of the various species. We are particularly interested in the map $F\left(\mathbf{x}_{0}\right) \doteq \psi_{\bar{t}}\left(\mathbf{x}_{0}\right)$, i.e., the evolution of the species system over one period. In the following we will prove some results about the stability of the system under one iteration of the map $F$. For simplicity here we have considered the case $n=2$ but the arguments will work for general $n$ as well. Without loss of generality, we assume that $g_{1}>g_{2}$. We also assume that initially, both types consume glucose $\left(g(0)>g_{1}\right)$.

We first develop some bounds on $x_{i}$. First observe that for all $t \in[0, \bar{t})$ we have $g(t) \leq g(0)$ and $a(t) \leq a(0)$. From here on we will simplify the notation as $A=\gamma g(0)-d$. Assume that $g(0)>a(0)$, then we have for each $i=1,2$

$$
\begin{aligned}
& x_{i}(t) \leq x_{i}(0) \exp [t A] \\
& x_{i}(t) \geq x_{i}(0) e^{-d t} .
\end{aligned}
$$

A simple lower bound on $g$ now follows

$$
g(t) \geq \hat{g}_{1}(t)=g(0) \exp \left[-\frac{x_{1}(0)+x_{2}(0)}{A}\left(e^{t A}-1\right)\right] .
$$

Simple calculations reveal that

$$
\hat{g}_{1}^{-1}(x)=\frac{1}{A} \log \left(1-\frac{A}{x_{1}(0)+x_{2}(0)} \log \frac{x}{g(0)}\right) .
$$

We can thus define the switching times under the lower bound $\hat{g}_{1}$,

$$
\tau_{i}^{(1)}=\hat{g}_{1}^{-1}\left(g_{i}\right)
$$

We now will use (3.4) to show that $\hat{x}=x_{1}(0)+x_{2}(0)$ cannot get too small. In particular we want to show the following result.

LEMMA 3.1. Assume that $\gamma \min \left(g_{1}, g_{2}\right)>d$ then for any $T>0$ there is an $\varepsilon>0$ such that if $\hat{x} \leq \varepsilon$ then $x_{1}(T)+x_{2}(T)>\hat{x}$.

Proof. We consider the lower bound first

$$
\dot{x}_{i}(t)=\left(\gamma S_{i}(g) g-d\right) x_{i} \geq\left(\gamma S_{i}\left(\hat{g}_{1}\right) \hat{g}_{1}-d\right) x_{i}
$$


Thus

$$
\begin{aligned}
x_{i}(T) & \geq x_{i}(0) e^{-d T} \exp \left[g(0) \gamma \int_{0}^{\tau_{i}^{(1)} \wedge T} \exp \left(-\frac{\hat{x}}{A}\left(e^{A t}-1\right)\right) d t\right] \\
& \geq x_{i}(0) e^{-d T} \exp \left[\gamma\left(\tau_{i}^{(1)} \wedge T\right) \hat{g}_{1}\left(\tau_{i}^{(1)} \wedge T\right)\right] \\
& \geq x_{i}(0) e^{-d T} \exp \left[\gamma\left(\tau_{i}^{(1)} \wedge T\right) g_{i}\right] .
\end{aligned}
$$

From the formula for $\tau_{i}^{(1)}$ we see that if

$$
\hat{x} \leq \frac{A \log \left(g(0) / g_{1}\right)}{e^{A T}-1}
$$

then $\tau_{i}^{(1)} \geq T$. The bound then follows from the assumption of the lemma.

We next will establish that if $x_{i}(0)$ is sufficiently large then $x_{i}(T)<x_{i}(0)$.

LEMMA 3.2. For any $T>0$ there is $K<\infty$ such that if $x_{i}(0)>K$ then $x_{i}(T)<x_{i}(0)$.

Proof. For each $i$ define the time when species $i$ switches from glucose to acetate

$$
\tau_{i}=\inf \left\{t>0: g(t) \leq g_{i}\right\}
$$

From the system of differential equations we have that for $i \in\{1, \ldots, n\}$

$$
\begin{aligned}
& x_{i}(t)=x_{i}(0) e^{-d t} \exp \left[\gamma \int_{0}^{\tau_{i} \wedge t} g(s) d s+\alpha \int_{\tau_{i} \wedge t}^{t} a(s) d s\right] \\
& g(t)=g(0) \exp \left[-\sum_{j=1}^{n} \int_{0}^{\tau_{j} \wedge t} x_{j}(s) d s\right] \\
& a(t)=a(0) \exp \left[-\sum_{j=1}^{n} \int_{\tau_{j} \wedge t}^{t} x_{j}(s) d s\right] .
\end{aligned}
$$

To simplify notation set $\hat{\gamma}=\gamma g(0)$ and $\hat{\alpha}=\alpha a(0)$. If we plug in the formulas for $g$ and $a$ into the formula for $x_{i}$ and observe that each $x_{i}$ is monotonically increasing we get

$$
\begin{aligned}
\frac{x_{i}(t)}{x_{i}(0)} e^{d t} & =\exp \left[\hat{\gamma} \int_{0}^{\tau_{i} \wedge t} \exp \left(-\sum_{j=1}^{n} \int_{0}^{\tau_{j} \wedge s} x_{j}(r) d r\right) d s+\hat{\alpha} \int_{\tau_{i} \wedge t}^{t} \exp \left(-\sum_{j=1}^{n} \int_{\tau_{j} \wedge s}^{s} x_{j}(r) d r\right) d s\right] \\
& \leq \exp \left[\hat{\gamma} \int_{0}^{\tau_{i} \wedge t} \exp \left(-\sum_{j=1}^{n} x_{j}(0)\left(\tau_{j} \wedge s\right)\right) d s+\hat{\alpha} \int_{\tau_{i} \wedge t}^{t} \exp \left(-\sum_{j=1}^{n} x_{j}(0)\left(s-\tau_{j}\right)^{+}\right) d s\right] .
\end{aligned}
$$

First observe that 


$$
\int_{0}^{\tau_{i} \wedge t} \exp \left(-\sum_{j=1}^{n} x_{j}(0)\left(\tau_{j} \wedge s\right)\right) d s \leq \int_{0}^{\tau_{i} \wedge t} e^{-x_{i}(0) s} d s \leq \frac{1}{x_{i}(0)}
$$

When considering the second integral in (3.5) it suffices to assume that $\tau_{i}<t$, thus consider

$$
\int_{\tau_{i}}^{t} \exp \left[-\sum_{j=1}^{n} x_{j}(0)\left(s-\tau_{j}\right)^{+}\right] d s \leq \int_{\tau_{i}}^{t} \exp \left(-x_{i}(0)\left(s-\tau_{j}\right)\right) d s \leq \frac{1}{x_{i}(0)} .
$$

We can plug the previous two displays into (3.5) and see that

$$
\frac{x_{i}(t)}{x_{i}(0)} \leq \exp \left[\frac{\hat{\gamma}+\hat{\alpha}}{x_{i}(0)}-d t\right]
$$

and the Lemma follows immediately.

These results provide some conditions for stability of the single-period dynamics of our model. In particular we show a sufficient condition on the relationship between the model parameters (growth rate on glucose consumption, acetate switching thresholds and the death rate) such that the total population will not go extinct. On the other hand, we also show that the population cannot blow up; in particular there exists a threshold population size above which the population of any given type will decrease.

\subsection{Proof of Coexistence Under Resampling Conditions}

In this section we consider coexistence under the assumption that resampling occurs. Specifically, suppose that (3.1-3.3) describe the dynamics during any given period, but that the total population size is the same at the beginning of each period. The initial values of $x_{1}, x_{2}$ at the beginning of each period are set such that the ratio $x_{1} / x_{2}$ is the same as at the end of the previous period (i.e., the population is resampled according to the relative abundances of each type). The definition of coexistence under resampling conditions is slightly more relaxed than without resampling, since here it allows for the scenario where both populations decrease during each period while preserving their relative frequencies within the total population. We note that resampling was utilized in the experimental study [13] of speciation in E. coli. In the following we provide a proof that it is possible to find a suitable resource influx level at the beginning of each period such that coexistence under resampling is possible. 
Consider the case when there are two types. Without loss of generality, suppose $g_{1}>g_{2}$. We will suppose that $g(0)>g_{1}$. This condition is not necessary for our results about coexistence but it is clearer to discuss this scenario than to consider all possible cases.

The glucose level $g$ never increases in the interval $0<t<T$. There are three possible scenarios; the glucose level is always greater than $g_{1}$, the glucose level falls to a level between $g_{1}$ and $g_{2}$, and the glucose level falls to a level below $g_{2}$. Let $t_{1}$ denote the time at which $g$ reaches $g_{1}$ or, if it never reaches $g_{1}$, let it be equal to $T$. Similarly let $t_{2}$ denote the time at which $g$ reaches $g_{2}$, or, if it never reaches $g_{2}$, let it be equal to $T$. The glucose level decreases in the interval $0<t<t_{2}$ and remains constant in the interval $t_{2}<t<T$. The acetate level remains constant in the interval $0<t<t_{1}$ and decreases in the interval $t_{1}<t<T$.

In the interval $0<t<t_{1}$ we have the following system of differential equations

$$
\begin{aligned}
\dot{x}_{1} & =(g \gamma-d) x_{1} \\
\dot{x}_{2} & =(g \gamma-d) x_{2} \\
\dot{g} & =-g\left(x_{1}+x_{2}\right) \\
\dot{a} & =0 .
\end{aligned}
$$

There are a number of important observations we can make about this system. For example, if $g(0)>d / \gamma$, the populations grow initially. This is the case in the experimental conditions. If, moreover, $g_{1}>d / \gamma$, then the first population switches to consuming acetate before the glucose level is small enough to cause the populations to decrease. The most important observation in terms of coexistence is that the ratio $x_{1}(t) / x_{2}(t)$ remains constant throughout the interval.

In the interval $t_{1}<t<t_{2}$ we have two uncoupled two-dimensional systems in $x_{1}$ and $a$ and in $x_{2}$ and $g$. They are

$$
\begin{aligned}
\dot{x}_{1} & =(a \alpha-d) x_{1} \\
\dot{a} & =-a x_{1}
\end{aligned}
$$

and

$$
\begin{aligned}
\dot{x}_{2} & =(g \gamma-d) x_{2} \\
\dot{g} & =-g x_{2}
\end{aligned}
$$

The initial conditions for this system are the values of $x_{1}, x_{2}, g$ and $a$ at time $t_{1}$ where $a\left(t_{1}\right)=a(0)$ since $a$ wasn't consumed at all in the interval $0 \leq t<t_{1}$. Notice that the percentage growth rate of $x_{1}$ is greater than the percentage growth rate of $x_{2}$ if and only if $a(t) \alpha>g(t) \gamma$. In particular, if $a(0) \alpha>g_{1} \gamma$, then the first population gets a boost as a result of switching to consumption of acetate. However, this boost may not continue throughout the whole interval. For example, if $x_{2}\left(t_{1}\right)<x_{1}\left(t_{1}\right)$ 
then, at least initially, the acetate will be consumed faster than the glucose, so it's quite possible that at some time $t, a(t) \alpha$ will fall below $g(t) \gamma$ and $x_{1}$ will grow more slowly than $x_{2}$.

In the interval $t_{2}<t<T$, we have

$$
\begin{aligned}
\dot{x}_{1} & =(a \alpha-d) x_{1} \\
x_{2} & =(a \alpha-d) x_{2} \\
\dot{a} & =-a\left(x_{1}+x_{2}\right) \\
\dot{g} & =0 .
\end{aligned}
$$

This system is similar to that in the first interval except the E. coli are consuming acetate instead of glucose. In particular, the ratio $x_{1}(t) / x_{2}(t)$ remains constant throughout the interval.

The two populations coexist if their relative abundances remain bounded away from 0 and away from $\infty$ for a long period of time. Since non-zero populations cannot be annihilated or become infinite in finite time in this model, a sufficient condition for coexistence is that $x_{1}(0) / x_{2}(0)$ be (very nearly) equal to $x_{1}(T) / x_{2}(T)$. This is trivially true in the case when $t_{1}=t_{2}=T$. But this case doesn't capture the essence of sympatric speciation, since the two populations behave identically under the environmental conditions in this case. The lemma below shows for all parameter values there are initial conditions under which we have equality of these ratios and under which the two populations exhibit a differentiated response to the environment. A closer examination of the proof of the lemma actually reveals a submanifold of co-dimension one in which this occurs. Since coexistence only requires the ratios to be very nearly equal, it follows there is a whole open set in the space of initial conditions in which we have coexistence.

LemMA 3.3. Given parameter values $\alpha, \gamma, d, g_{1}, g_{2}$, and $T$, there exist initial conditions $a(0), g(0), x_{1}(0)$, and $x_{2}(0)$ such that $t_{1}<t_{2} \leq T$ and

$$
\frac{x_{1}(0)}{x_{2}(0)}=\frac{x_{1}(T)}{x_{2}(T)}
$$

Proof. Let $\alpha, \gamma, d, g_{1}, g_{2}$, and $T$ be given. Since for any initial conditions,

$$
\frac{x_{1}(0)}{x_{2}(0)}=\frac{x_{1}\left(t_{1}\right)}{x_{2}\left(t_{1}\right)} \quad \text { and } \quad \frac{x_{1}\left(t_{2}\right)}{x_{2}\left(t_{2}\right)}=\frac{x_{1}(T)}{x_{2}(T)}
$$

it suffices to show the existence of initial conditions under which $t_{1}<t_{2} \leq T$ and

$$
\frac{x_{1}\left(t_{1}\right)}{x_{2}\left(t_{1}\right)}=\frac{x_{1}\left(t_{2}\right)}{x_{2}\left(t_{2}\right)}
$$


Given $t_{1}$, whether or not equation (3.18) is satisfied is completely determined by the values of the variables at time $t_{1}$. By definition of $t_{1}, g\left(t_{1}\right)=g_{1}$. To prove the result we choose the value of $t_{1}$ and show that we can then choose $x_{1}\left(t_{1}\right), x_{2}\left(t_{1}\right)$ and $a\left(t_{1}\right)=a(0)$ to satisfy equation (3.18). We can then run the system of differential equations (3.6)-(3.9) backwards in time from $t_{1}$ to get the corresponding initial values of the variables at time 0 .

The choice of $t_{1}$ is arbitrary. For example, we can choose $t_{1}=T / 2$. The value of $x_{2}\left(t_{1}\right)$ is also arbitrary. In the experimental setup this would be large enough that $t_{2}<T$ but this is not essential for our result. In the interval $t_{1}<t<t_{2}$ the value of $g$ is determined by the two-dimensional system of differential equations (3.12)-(3.13) in $x_{2}$ and $g$ with initial conditions $x_{2}\left(t_{1}\right)$ and $g\left(t_{1}\right)=g_{1}$. This means the values of $t_{1}$ and $x_{2}\left(t_{1}\right)$ determine the values of $t_{2}$ and the ratio

$$
r \doteq \frac{x_{2}\left(t_{2}\right)}{x_{2}\left(t_{1}\right)} .
$$

To prove we can find $x_{1}\left(t_{1}\right)$ and $a\left(t_{1}\right)$ such that equation (3.18) is true, we show we can find values where

$$
\frac{x_{1}\left(t_{1}\right)}{x_{2}\left(t_{1}\right)}>\frac{x_{1}\left(t_{2}\right)}{x_{2}\left(t_{2}\right)}
$$

and other values where

$$
\frac{x_{1}\left(t_{1}\right)}{x_{2}\left(t_{1}\right)}<\frac{x_{1}\left(t_{2}\right)}{x_{2}\left(t_{2}\right)}
$$

By continuity of these ratios in the initial conditions, it follows there are values where equation (3.18) is true.

For inequality (3.19), $x_{1}\left(t_{1}\right)$ can have any value and $a\left(t_{1}\right)$ should be small enough that $a\left(t_{1}\right)<g_{2} \gamma / \alpha$. In this case, for all $t$ between $t_{1}$ and $t_{2}$,

$$
a(t) \alpha-d<a\left(t_{1}\right) \alpha-d<g_{2} \gamma-d<g(t) \gamma-d .
$$

In other words, the percentage growth rate of $x_{1}$ is always less than the percentage growth rate of $x_{2}$, so inequality (3.19) is satisfied.

For inequality (3.20), choose $a\left(t_{1}\right)$ large enough that $a\left(t_{1}\right) \alpha-d>0$ and

$$
\exp \left[\left(\frac{a\left(t_{1}\right) \alpha}{2}-d\right)\left(t_{2}-t_{1}\right)\right]>r .
$$

Define

$$
A \doteq \exp \left[\left(a\left(t_{1}\right) \alpha-d\right)\left(t_{2}-t_{1}\right)\right]
$$


Then choose $x_{1}\left(t_{1}\right)$ small enough that

$$
\exp \left[-x_{1}\left(t_{1}\right) A\left(t_{2}-t_{1}\right)\right]>\frac{1}{2} .
$$

Since $a$ is decreasing throughout the interval $t_{1}<t<t_{2}$, its value at the beginning of the interval provides an upper bound on the growth rate of $x_{1}$ and hence on $x_{1}$. More specifically,

$$
\dot{x_{1}}=(a \alpha-d) x_{1}<\left(a\left(t_{1}\right) \alpha-d\right) x_{1}
$$

so

$$
x_{1}(t)<x_{1}\left(t_{1}\right) \exp \left[\left(a\left(t_{1}\right) \alpha-d\right)\left(t-t_{1}\right)\right] .
$$

Since $a\left(t_{1}\right) \alpha-d>0$, the function on the right is increasing, so

$$
x_{1}(t)<x_{1}\left(t_{1}\right) A .
$$

This upper bound for $x_{1}$ then provides an upper bound for the rate at which $a$ is decreasing and therefore provides a lower bound for the value of $a$. More specifically,

$$
\dot{a}=-a x_{1}>-a x_{1}\left(t_{1}\right) A
$$

so

$$
a(t)>a\left(t_{1}\right) \exp \left[-x_{1}\left(t_{1}\right) A\left(t-t_{1}\right)\right]>a\left(t_{1}\right) \exp \left[-x_{1}\left(t_{1}\right) A\left(t_{2}-t_{1}\right)\right]>\frac{a\left(t_{1}\right)}{2}
$$

by our choice of $x_{1}\left(t_{1}\right)$. This lower bound for the value of $a$ then gives us a lower bound for the value of $x_{1}$. More specifically,

$$
\dot{x_{1}}=(a \alpha-d) x_{1}>\left(\frac{a\left(t_{1}\right) \alpha}{2}-d\right) x_{1}
$$

so

$$
x_{1}\left(t_{2}\right)>x_{1}\left(t_{1}\right) \exp \left[\left(\frac{a\left(t_{1}\right) \alpha}{2}-d\right)\left(t_{2}-t_{1}\right)\right]
$$

Thus

$$
\frac{x_{1}\left(t_{2}\right)}{x_{1}\left(t_{1}\right)}>r
$$

and we have inequality (3.20). 


\subsection{Computational Exploration of Coexistence in a Two-Type System}

We considered the situation of a single 'fast-switching' (FS) and 'slow-switching' (SS) type. One particular question of interest is: Given a fixed environmental influx of both food sources every twenty-four hours, what range in relative differences of metabolic benefit between these food sources can give rise to coexistence? In other words, we explore the region of the $\gamma-\alpha$ parameter space to locate regions of coexistence. In this section we return to the model (3.1-3.3) without resampling.

Figure 4a shows a map of the frequency of fast switchers in the population after 1500 days, for a range of $\gamma$ and $\alpha$ values. Regions of intermediate color (not dark
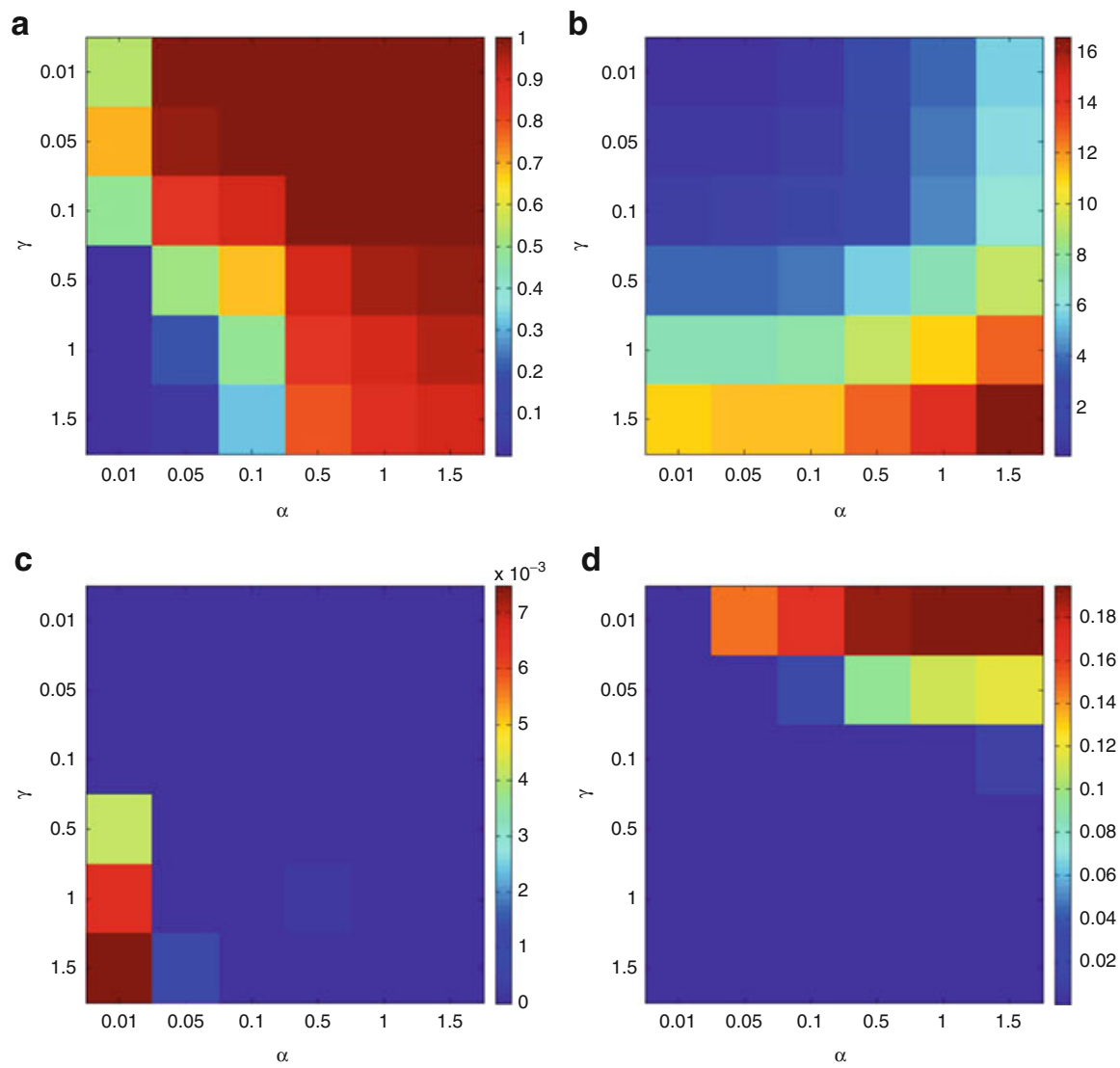

Fig. 4 Computational analysis of coexistence in system (3.1)-(3.3) with two types $(n=2)$ : an SS with $g_{1}=0.01$ and an FS with $g_{2}=0.1$. Parameters $\gamma, \alpha$ are varied, all other parameters held constant: $d=0.01, k=50$ and initial influx of 2 units glucose and 1 unit acetate every 24 hours. All plots display the state of the system after 1500 days. (a) Percentage of population comprised of fast-switchers, (b) Total population size, (c) Percentage change in FS population size in last 24 hours, (d) Percentage change in SS population size in last 24 hours. 
red or blue) correspond to parameter regions where steady state coexistence occurs between FS and SS. Figure $4 \mathrm{~b}$ shows the total population size of fast and slow switchers combined; note that when both $\gamma$ and $\alpha$ are low, the population declines overall. As demonstrated by the percentage change in the population sizes of fast and slow switchers (Figures $4 \mathrm{c}$ and $\mathrm{d}$ ), the system in the coexistence regions has reached a steady state by this time. Other regions of higher than negligible percentage change correspond to regions where the respective population sizes are very close to zero. From these plots we observe that coexistence between FS and SS exists in regions of parameter space where $\gamma$ is relatively close to $\alpha$ or slightly larger. For large relative values of $\alpha$, the slow-switcher population tends to die out. This can be attributed to the fact that large $\alpha$ corresponds to a large rate of growth of both species while using acetate; since the fast switchers switch to acetate consumption more quickly, they will tend to win out over the SS. Similarly, for large relative values of $\gamma$, the FS population tends to die out; since the SS are able to consume glucose for longer they will benefit more from higher $\gamma$. We also note that we expect that $\gamma \geq \alpha$ in real life applications, since glucose is the preferred, more efficient food source. Therefore, if constrained to this portion of the parameter space, we would expect either coexistence (if $\gamma$ is close to $\alpha$ ) or for the fast switchers to die out.

We also examined the impact of the initial influx values of glucose and acetate each day on the coexistence behavior of the system. Clearly if $\gamma g_{0}-d<0$ and $\alpha a_{0}-d<0$ hold, then the total population will tend to zero over time. Outside of this region, Figure 5 demonstrates how the long-time behavior of the system varies as the daily influx values of glucose and acetate are varied, keeping all other parameters constant. Figure 5a shows the steady state level of fast switchers in the population; we observe that as the acetate and glucose influxes increase, the fast switchers reach higher population levels. Figure $5 \mathrm{~b}$ shows the steady state level of slow switchers in the population; we observe that as the glucose influx increases and as the acetate influx decreases, the slow switchers reach higher population levels. In other words, both populations benefit from an increase in the primary food source (glucose). However the fast switchers benefit from an increase in the secondary food source since they are able to consume acetate first. The slow switchers benefit from a decrease in acetate availability, since that results in slower growth of the FS population which allows for more glucose for the SS population. We also note that, as observed in Figure 5c, the total population size is most sensitive to changes in the primary food source (glucose) and less sensitive to changes in acetate influx.

\subsection{Quasiperiodic and Random Environments}

The periodicity of the environment is often subject to noise, both in terms of period and amplitude of resource influx, in natural settings. We next investigated the impact of noise in the environment on the system. Figure 6 demonstrates an example of 

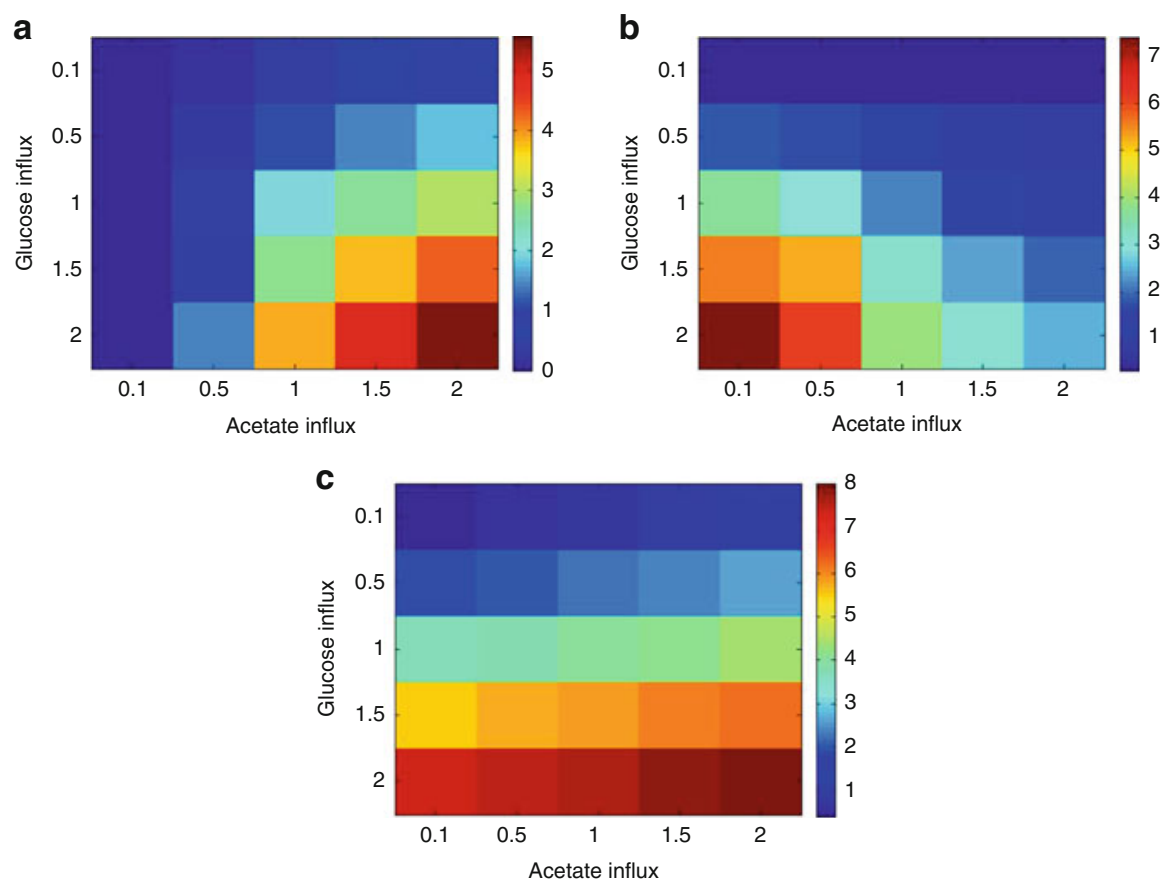

Fig. 5 Computational analysis of coexistence in system (3.1)-(3.3) with two types $(n=2)$ : an SS with $g_{1}=0.01$ and an FS with $g_{2}=0.1$. The daily influx of glucose and acetate are varied, all other parameters held constant: $d=0.01, k=50, \alpha=0.1, \gamma=1$ and influx of glucose and acetate occurs every 24 hours. All plots display the state of the system after 1500 days when the system has reached steady state. (a) FS population size, (b) SS population size, (c) Total population size.

the impact of a randomized cycle length (uniformly distributed on an interval of 6 around a 24-hr mean) or stochastic influx of glucose and acetate levels on the long-term behavior of the system. Note that the threshold glucose levels at which FS and SS types switch to consuming acetate are deterministic even during these investigations on quasiperiodic/random environments. We observe that both the value of the steady state population sizes and the time to reach those sizes are not impacted significantly by these variations. In fact, Figure $6 \mathrm{~d}$ reproduces the same parameter space plot investigating the coexistence region as in Figure 4a, now incorporating stochastic cycle lengths as well as influx values of glucose and acetate; we observe that the plots are essentially identical indicating a robustness of these behaviors to these types of noise in the environment. 

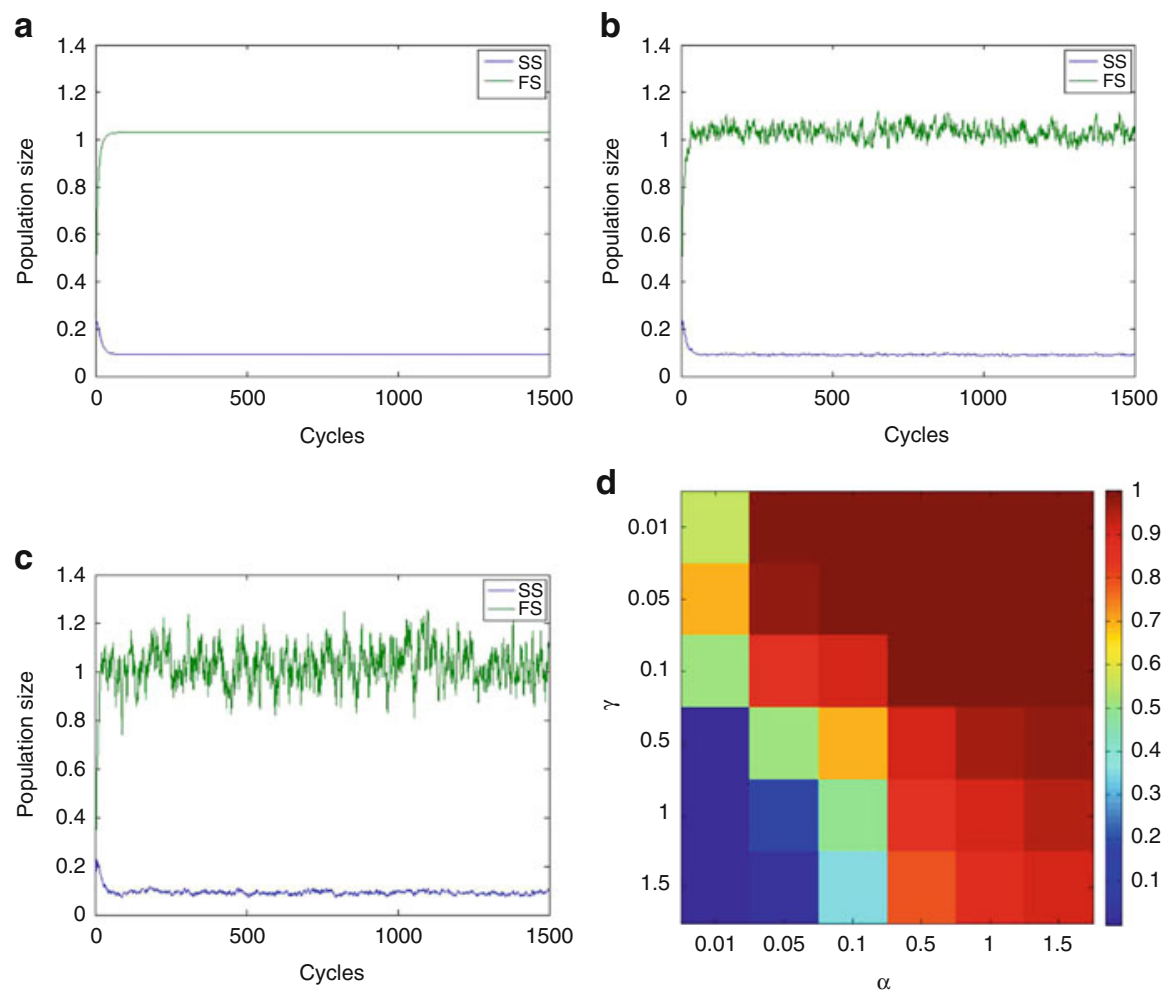

Fig. 6 Effects of quasiperiodic environmental fluctuation on system (3.1)-(3.3) with two types $(n=2)$ : an SS with $g_{1}=0.01$ and an FS with $g_{2}=0.1$, compared to deterministic 24-hr cycle lengths. All other parameters held constant: $d=0.01, k=50, \alpha=0.1, \gamma=0.1$. (a) Long-term behavior of FS and SS over 1500 cycles for deterministic cycle length of 24 hours, and deterministic influx of glucose (2 units) and acetate (1 unit). (b) Long-term behavior of FS and SS over 1500 cycles for stochastic cycle length $T \sim U([21,27])$, and deterministic influx of glucose (2 units) and acetate (1 unit) (c) Long-term behavior of FS and SS over 1500 cycles for deterministic cycle length of 24 hours and stochastic influx conditions of glucose $U([1,3])$ and acetate $U([0.5,1.5])$, (d) Parameter space map corresponding to 4 a, for quasiperiodic random environment: random glucose influx $U([1,3])$, acetate influx $U([0.5,1.5])$, and cycle length $T \sim$ $U([21,27])$.

\section{Spatial Interpretation of the Model}

A cellular automata model of the system was subsequently developed to investigate what spatial effects could be observed. Previously, agent based modeling has been used to show the possibility of speciation [14], although this model was focused on sexually reproducing species. The model was developed in Netlogo [28] using the same parameters and equations as were used in the ODE system, 
equations (3.1-3.3). These equations describe the change in cell types over time as well as the rate at which the nutrients are used. These relationships are maintained in the Netlogo model.

The present model was initialized with a population of cells whose switch point $g_{i}$ ranged from 0 to 0.1 . The cells are initially randomly distributed across the domain and are randomly assigned a switch point $\left(g_{i}\right)$. The cells were then grouped into 20 categories by their threshold value. The model was run for 2000 iterations, with nutrient replenishment occurring every 20 time steps. The nutrients were allowed to diffuse within the environment. $E$. coli cells were only allowed to consume nutrients from their immediate locale (patch). Cells reproduced asexually, assuming the local environment was not too crowded (neighborcount $<8$ ), by creating a new cell, identical to themselves. The new cell was allowed to search the immediate area (Moore neighborhood) for the least crowded patch to call home, thereby spreading out the resource demand within the model domain. So, the cells do not move around the domain, but the progeny are able to spread out over time.

With this version of the model, we no longer have a well-mixed environment. Both the cells and the nutrients vary spatially. The cells remain fixed in their location, but the offspring are placed in surrounding locations. The nutrients, glucose and acetate, diffuse through the environment and are used up locally. Netlogo has a built-in function for diffusion that is used for this model. In this case, we specify that $50 \%$ of the resource is distributed to the neighboring patches at each time step.

The simulations produce results similar to the ODE model, with a preference for the slow switchers when parameter values $\gamma$ and $\alpha$ are in the appropriate range, see Figure 7. Furthermore, we see the dominant persistence of only a few species. Therefore, adding a spatial component does not interfere with the underlying dynamics of the system. This can be viewed as further evidence that the speciation is sympatric. There is no geographic component to the evolutionary dynamics.
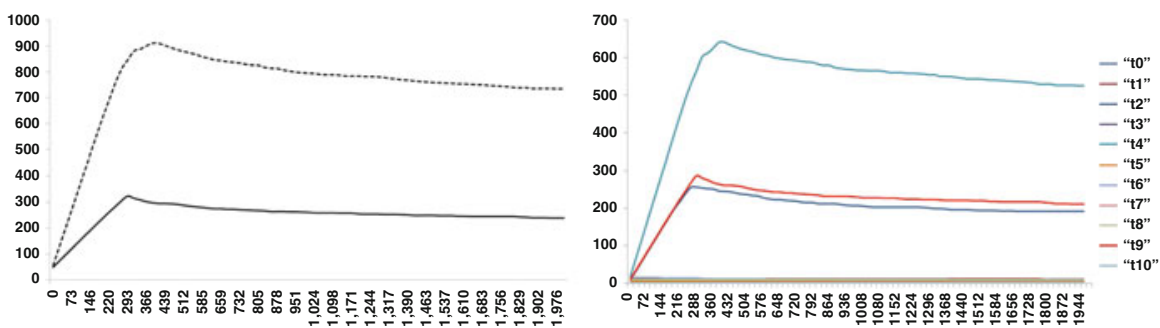

Fig. 7 Typical dynamics of system (3.1-3.3) implemented as a cellular automata model, where 20 types were randomly created by assigning their value $g_{i}$ from a uniform distribution between 0 and 0.1. In the left panel, the cumulative abundances of slow switchers (dashed) and fast switchers (solid) are shown as a function of the iteration number. In the right panel, the individual categories are plotted. The slow switch types are shown in blue and the fast switch are in red. The parameters of the system are: $n=20, \bar{t}=20$ (resampling time), $a_{0}=g_{0}=1$ (the initial values of $a$ and $g$ at the start of each iteration), $a=10, \gamma=1, \alpha=0.1, d=0.1$. 


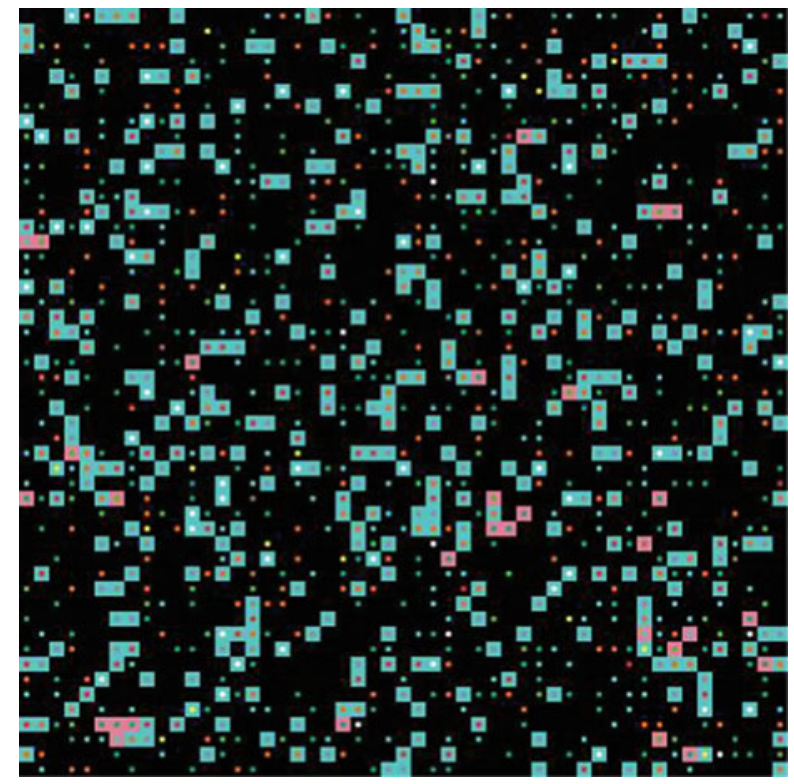

Fig. 8 Clustering of slow switchers (blue) and fast switchers (pink). The parameters of the system are: $n=20, \bar{t}=20$ (resampling time), $a_{0}=g_{0}=1$ (the initial values of $a$ and $g$ at the start of each iteration), $a=10, \gamma=1, \alpha=0.1, d=0.1$.

One interesting phenomenon that did emerge from the investigation of the spatially dynamic version of the model is that the slow switcher phenotypes show a greater affinity for clustering than the fast switchers. Figure 8 shows a plot with shaded patches when cells of similar phenotypes are neighboring. The slow switch phenotypes are shown in blue and the fast switch phenotypes are shown in red. Even though the overall number of cells in the two categories are close (within $10 \%$ ), the amount of clustering seen in the slow switchers is much larger (405 slow switchers were flagged as clustered compared to 30 fast switchers flagged as clustered.) We hypothesize that this is due to the nature of the resource usage. Since the fast switchers must consume a larger volume of acetate to maintain their viability, surviving fast switchers must remain more sparsely distributed.

\section{Summary}

In this work, we investigated the phenomenon of speciation driven by periodic or quasiperiodic fluctuations in resource abundances. We first developed a mathematical model of an experimentally observed phenomenon [13], in which $E$. coli exhibited sympatric diversification in a periodically replenished environment with two carbon-sources: glucose and acetate. In these experiments, two divergent 
phenotypes emerged, which differed in the threshold at which they switched from glucose consumption to acetate consumption. To investigate this phenomenon, we first formulated a system of differential equations to describe the evolution of $n$ types of E. coli, which differ in their glucose threshold for acetate switch. In particular, for each phenotype we explicitly modeled the level of activity of the acetate dissimilation pathway, which is stimulated by the glucose level. This model was observed to replicate the experimental outcome and results in coexistence behavior between two phenotypes - a fast-switcher and a slow-switcher.

Next, we developed a simplified model that retained only the features essential in describing the phenomenon of speciation into phenotypes with divergent metabolic behavior in the context of a quasiperiodic environment with multiple energy sources. Under this simplified model, we assume that each phenotype devotes a certain percentage of effort to consuming each energy source and this effort level is dependent upon the abundance of the primary energy source. This model was also demonstrated to result in coexistence among many phenotypes ranging from 'fast' to 'slow' switchers.

We investigated this simplified model both analytically and computationally. We analytically examined the conditions of stability for the single period system, and proved coexistence of species in the long-term dynamics; we also computationally investigated the coexistence properties of the periodically replenished system over many periods. We observed contained regions of coexistence in the parameter space of the two variables, $\gamma$ and $\alpha$, which describe the resulting growth of each type on glucose and acetate consumption, respectively. In particular, as $\gamma$ increases, $\alpha$ must also increase in order to preserve coexistence. For large values of $\alpha$, the slowswitcher population tends to die out, whereas for large values of $\gamma$, the fast-switcher population tends to die out. Since $\gamma$ represents growth on the primary resource, the inequality $\gamma \geq \alpha$ is expected to hold in real-life applications, and the model suggests that either coexistence would occur or fast-switchers would die out in a realistic scenario. We also studied the impact of modifying the abundance of resource influxes at the start of each period. We observed that while both populations benefit from an increase in the primary resource (glucose), only the slow-switchers benefit from increasing the secondary resource. Lastly we studied the impact of introducing stochasticity into the environment via both the length of the period and the size of the resource influxes; our coexistence regimes were robust to this noise.

A spatial version of the model was also developed to determine if spatial structure plays an important role in the coexistence properties of the system. We observed that the results are similar to the ODE model and that the spatial structure does not interfere with coexistence dynamics. Interestingly, we found that slow-switchers tend to cluster more than fast-switchers, and hypothesized that since the fastswitchers must consume more acetate after switching, they are less viable in clusters than slow-switchers.

Acknowledgements The author "Jasmine Foo" would like to acknowledge partial support through NSF DMS-1224362 and NSF DMS-1349724. The work described in this chapter is a result of a collaboration made possible by the IMA's WhAM! (Women in Applied Mathematics) Research Collaboration Workshop: Dynamical Systems with Applications to Biology and Medicine. 


\section{References}

1. R. A. Armstrong And R. McGehee, Competitive exclusion, American Naturalist, (1980), pp. 151-170.

2. M. Begon, J. HARPer, AND C. Townsend, Ecology, Blackwell Science, Oxford, 1996.

3. G. L. BUSH, Sympatric speciation in animals: new wine in old bottles, Trends in Ecology \& Evolution, 9 (1994), pp. 285-288.

4. A. M. DEAN, Protecting haploid polymorphisms in temporally variable environments, Genetics, 169 (2005), pp. 1147-1156.

5. U. Dieckmann And M. Doebeli, On the origin of species by sympatric speciation, Nature, 400 (1999), pp. 354-357.

6. M. Doebeli, Adaptive Diversification (MPB-48), Princeton University Press, 2011.

7. M. Doebeli AND U. DieCKMAnN, Evolutionary branching and sympatric speciation caused by different types of ecological interactions, The American Naturalist, 156 (2000), pp. S77-S101.

8. M. Doebeli And G. D. Ruxton, Evolution of dispersal rates in metapopulation models: branching and cyclic dynamics in phenotype space, Evolution, (1997), pp. 1730-1741.

9. J. FELSENSTEIN, Skepticism towards santa rosalia, or why are there so few kinds of animals?, Evolution, (1981), pp. 124-138.

10. M. L. Friesen, G. SAXer, M. Travisano, And M. Doebeli, Experimental evidence for sympatric ecological diversification due to frequency-dependent competition in escherichia coli, Evolution, 58 (2004), pp. 245-260.

11. S. A. Geritz, G. Mesze, J. MEtz, ET AL., Evolutionarily singular strategies and the adaptive growth and branching of the evolutionary tree, Evolutionary Ecology, 12 (1998), pp. 35-57.

12. S. Gourbiere AND J. Mallet, Has adaptive dynamics contributed to the understanding of adaptive and sympatric speciation?, Journal of Evolutionary Biology, 18 (2005), pp. 1201-1204.

13. M. D. Herron And M. Doebeli, Parallel evolutionary dynamics of adaptive diversification in escherichia coli, PLoS Biology, 11 (2013), p. e1001490.

14. R. HILSCHER, Agent-based models of competitive speciation $i$ : effects of mate search tactics and ecological conditions, Evolutionary Ecology Research, 7 (2005), pp. 943-971.

15. S. HsU, A competition model for a seasonally fluctuating nutrient, Journal of Mathematical Biology, 9 (1980), pp. 115-132.

16. G. E. HutCHInson, The paradox of the plankton, American Naturalist, (1961), pp. 137-145.

17. J. JOHANSSON AND J. RIPA, Will sympatric speciation fail due to stochastic competitive exclusion?, The American Naturalist, 168 (2006), pp. 572-578.

18. P. A. Johnson, F. Hoppensteadt, J. J. Smith, AND G. L. Bush, Conditions for sympatric speciation: a diploid model incorporating habitat fidelity and non-habitat assortative mating, Evolutionary Ecology, 10 (1996), pp. 187-205.

19. A. S. KondRASHOV, Multilocus model of sympatric speciation. iii. computer simulations, Theoretical Population Biology, 29 (1986), pp. 1-15.

20. O. LEIMAR, The evolution of phenotypic polymorphism: randomized strategies versus evolutionary branching, The American Naturalist, 165 (2005), pp. 669-681.

21. T. NAMBA AND S. TAKAHASHI, Competitive coexistence in a seasonally fluctuating environment ii. multiple stable states and invasion success, Theoretical Population Biology, 44 (1993), pp. 374-402.

22. M. L. Rosenzweig, Competitive speciation, Biological Journal of the Linnean Society, 10 (1978), pp. 275-289.

23. J. M. Smith, Sympatric speciation, American Naturalist, (1966), pp. 637-650.

24. C. C. Spencer, M. Bertrand, M. Travisano, and M. Doebeli, Adaptive diversification in genes that regulate resource use in escherichia coli, PLoS Genetics, 3 (2007), p. e15. 
25. C. C. Spencer, G. SAXer, M. Travisano, And M. Doebeli, Seasonal resource oscillations maintain diversity in bacterial microcosms, Evolutionary Ecology Research, 9 (2007), p. 775.

26. F. M. STEWART AND B. R. LeVIn, Partitioning of resources and the outcome of interspecific competition: a model and some general considerations, American Naturalist, (1973), pp. 171-198.

27. D. WAXMAN AND S. GAVRILETS, 20 questions on adaptive dynamics, Journal of Evolutionary Biology, 18 (2005), pp. 1139-1154.

28. U. WILENSKY, NetLogo, http://ccl.northwestern.edu/netlogo/, Center for Connected Learning and Computer-Based Modeling, Northwestern University. Evanston, IL, 1999.

29. A. Wolfe, The acetate switch, Microbiol Mol Biol Rev, 69 (2005), pp. 12-50. 